\title{
Multi-scale Local Binary Pattern Histograms for Face Recognition
}

\author{
Chi-Ho Chan, Josef Kittler, and Kieron Messer \\ Centre for Vision, Speech and Signal Processing, \\ University of Surrey, United Kingdom \\ \{c.chan, j.kittler,k.messer\}@surrey.ac.uk
}

\begin{abstract}
A novel discriminative face representation derived by the Linear Discriminant Analysis (LDA) of multi-scale local binary pattern histograms is proposed for face recognition. The face image is first partitioned into several non-overlapping regions. In each region, multi-scale local binary uniform pattern histograms ${ }^{1}$ are extracted and concatenated into a regional feature. The features are then projected on the LDA space to be used as a discriminative facial descriptor. The method is implemented and tested in face identification on the standard Feret database and in face verification on the XM2VTS database with very promising results.
\end{abstract}

\section{Introduction}

Nowadays, face identification and verification are an integral part of the infrastructure needed for diverse business and security sectors. However, the performance of most current systems is detrimentally affected by two factors: 1) large variability in facial appearance of an individual due to changes in pose, expression and age, as well as ambient light condition and camera parameters, and 2) high dimensionality of the problem with a small sample size training. For tackling the above problems, one of the important strategies is to represent a face image by projecting it into a "good" feature space which captures the intrinsic attributes of face and simplifies the face manifolds of the raw image space. The most popular representations proposed for this purpose include Eigenface, Fisherface, Wavelets (Gabor and Haar).

Recently, the local binary pattern (LBP) operator, introduced by Ojala et.al [10], has gained reputation as a powerful and attractive texture descriptor showing excellent results in terms of accuracy and computational complexity in many empirical studies. The LBP method has already been applied for instance to visual inspection, image retrieval, motion detection, remote sensing, biomedical image analysis, and outdoor scene analysis. Ahonen et al. [9] applied a LBP representation to face recognition and achieved very good results on the FERET database. In their method, the face image is first partitioned into small regions from which LBP histograms are extracted and concatenated into a single, spatially enhanced feature histogram representing the local texture and global shape of face images. The recognition is performed using a nearest-neighbor classifier.

\footnotetext{
${ }^{1}$ This paper is for BBSPA competition.
} 
Later, Zhang et al. [2] identified two shortcomings of Ahonen's approach. First, the size of the feature space in Ahonen's method was limited by fixing the position and size of the local region. Second, the region weighting was manually optimized. Therefore, they proposed to use a boosting classifier [2][12] to select discriminative histograms from a pool which is obtained by extracting the LBP histograms by shifting and scaling a local window over pairs of intra-personal and inter-personal face images. Comparative studies with Ahonen's method on the FERET database FB protocol showed similar results in accuracy but as fewer regional histograms are used, the dimensionality of the representation space is lower. However, shifting and scaling the local window will result in an over-complete representation requiring a prohibitive amount of time for training. In addition, the accuracy is dependent on a predefined feature number or a predefined recognition rate, and thus is not optimal. Other interesting contributions include the work of Rodriguez and Marcel [14] who proposed a generative approach for face verification based on applying a LBP histogram as the face descriptor. Shan et al. [8] advocated the use of a linear discriminant analysis (LDA) classifier on LBP local histograms and showed that their results outperformed the Ahonen's method. However, the small size $(4 \times 8)$ of the local region for computing the histogram tends to degrade the accuracy in the presence of face localization errors. Also, our results show that the accuracy of directly applying LDA on the uniform LBP local histograms is better than for their method. The reason is the way the pattern labels are grouped as already mentioned by Ojala et.al [10] who pointed out that the histogram of uniform patterns provides better discrimination in comparison to the histogram of all individual patterns. The definition of uniform LBP will be introduced in the next section.

For multiresolution analysis, Zhang et al [11] proposed to apply LBP on Gabor wavelets, called MHLVP or LGBPHS, for face recognition and have very impressive results on the FERET database, but the computational complexity and the feature dimension are very high. In this paper, an alternative but a much simple discriminative descriptor containing the information from a multresolution analysis is proposed. The novel descriptor is formed by projecting the local information combined from multiple LBP operators into the LDA space. The recognition is performed by measuring the dissimilarity of the gallery and probe descriptors using normalized correlation. We evaluate the proposed algorithm on the Feret and XM2VTS databases and show that it exhibits superior performance in both face identification and face verification scenarios. The paper first introduces the basic LBP histogram and then describes the multi-scale LBP with LDA for face recognition. The experimental setup is then introduced and the results discussed.

\section{Face Description with Multi-scale LBP}

\subsection{Basic Uniform Local Binary Patterns}

The LBP operator, shown in Equation 1, a powerful texture measure with a lowcomputational complexity, extracts information which is invariant to local gray-scale variations of the image. During the LBP operation, the value of current pixel, $g_{c}$, is applied as a threshold to each of the neighbors, $\mathrm{g}_{\mathrm{p}}(\mathrm{p}=0, \ldots . \mathrm{P}-1)$ to obtain a binary 
number. A local binary pattern is obtained by first concatenating these binary numbers and then converting the sequence into the decimal number. Using circular neighborhoods and linearly interpolating the pixel values allows the choice of any radius, $\mathrm{R}$, and number of pixels in the neighborhood, $\mathrm{P}$, to form an operator.

$$
\begin{gathered}
L B P_{P, R}(x, y)=\sum_{p=0}^{P-1} s\left(g_{p}-g_{c}\right) 2^{p} \\
s(x)= \begin{cases}1 & x \geq 0 \\
0 & x<0\end{cases}
\end{gathered}
$$

where,

A subset of these $2^{\mathrm{P}}$ binary patterns, called uniform patterns, can be used to represent spot, flat area, edge and corner. The uniformity measure, $\mathrm{U}(\mathrm{x})$, presented in Equation 2 records the number of spatial transitions in the binary pattern, and the uniform pattern is a binary pattern which contains at most two bitwise transitions, i.e., $\mathrm{U}(\mathrm{x}) \leq 2$. The uniform pattern contains in total $(\mathrm{P}-1) \mathrm{P}+2$ binary patterns. It consists of two types of patterns, namely (P-1)P rotational patterns, such as edges and two non-rotational patterns, such as a bright spot or a flat area. Other patterns, where $\mathrm{U}(\mathrm{x})>2$, are regarded as non-uniform patterns. The uniform $\mathrm{LBP}$ operator, $\mathrm{LBP}_{\mathrm{P}, \mathrm{R}}^{\mathrm{u} 2}$, is defined as.

$$
L B P_{P, R}^{\mu 2}(x, y)=\left\{\begin{array}{cc}
I\left(L B P_{P, R}(x, y)\right) & \text { if } U\left(L B P_{P, R}\right) \leq 2, \\
& \mathrm{I}(\mathrm{z}) \in[0,(P-1) P+1] \\
(P-1) P+2 & \text { otherwise }
\end{array}\right.
$$

where, $U\left(L B P_{P, R}\right)=\left|s\left(g_{P-1}-g_{c}\right)-s\left(g_{0}-g_{c}\right)\right|+\sum_{p=1}^{P}\left|s\left(g_{p}-g_{c}\right)-s\left(g_{P-1}-g_{c}\right)\right|$

Superscript $\mathrm{u} 2$ shown in Equation 2 indicates that the definition relates to uniform patterns with a $U$ value of at most 2. If $U(x)$ is smaller than 2 , the current pixel will be labeled by an index function, $\mathrm{I}(\mathrm{z})$. Otherwise, it will be labeled as $(\mathrm{P}-1) \mathrm{P}+2$. The index function, $\mathrm{I}(\mathrm{z})$, containing $(\mathrm{P}-1) \mathrm{P}+2$ indices, is used to assign a particular index to each of the uniform patterns.

Some researchers used the LBP operator as one of the face normalization techniques [1] and then directly applied a LDA classifier to the LBP image. However, such an approach will fail in the presence of an image translation or even rotation. The histogram approach which first summarizes the LBP image statistically has been proposed to alleviate these problems. As keeping the information about the spatial relation of facial regions is very important for face recognition, the face image is first divided into several small non-overlapping regions of the same size. Uniform pattern histograms are computed over the regions and then concatenated into a single histogram representing the face image.

\subsection{Multi-scale Local Binary Patterns}

By varying the sampling radius, $\mathrm{R}$ and combining the LBP images, a multiresolution representation based on LBP, called multi-scale local binary patterns [10] can be obtained. This representation has been suggested for texture classification and the results reported for this application show that its accuracy is better than that of 
the single scale local binary pattern method. In general, this multiresolution representation can be realized in two ways. First, it can be accomplished by increasing the neighborhood size of the operator. Alternatively one can down-sample the original image with interpolation or low-pass filtering and then apply an LBP operator of fixed radius. However, the general problem associated with the multiresolution analysis is the high dimensionality of the representation combined with the small training sample size. It limits the total number of LBP operators to at most of 3 . One of the approaches [13] is to employ a feature selection technique to minimize redundant information. We propose another method which achieves dimensionality reduction by feature extraction.

\subsection{Our Approach}

In our approach, we combine the multi-scale local binary pattern representation with Linear Discrminant Analysis, LDA. Uniform local binary pattern operators at R scales are first applied to a face image. This generates a grey level code for each pixel at every resolution. The resulting LBP images, shown in Fig. 1, are cropped to the same size and divided into non-overlapping sub-regions, $\mathrm{M}_{0}, \mathrm{M}_{1}, . . \mathrm{M}_{\mathrm{J}-1}$. The regional pattern histogram for each scale is computed based on Equation 3.

$$
H_{P, r, j}^{\mu 2}(i)=\sum_{\left(x^{\prime}, y^{\prime}\right) \in M_{j}} B\left(L B P_{P, r}^{\mu 2}\left(x^{\prime}, y^{\prime}\right)=i\right)
$$

where, $\quad i \in[0,(P-1) P+2), r \in[1, R], j \in[0, J)$, and $B(x)=\left\{\begin{array}{ll}1 & \text { when } x=0 \\ 0 & \text { otherwise }\end{array}\right.$.

$\mathrm{B}(\mathrm{x})$ is a Boolean indicator. The set of histograms computed at different scales for region, $\mathrm{M}_{\mathrm{j}}$, provides regional information. By concatenating these histograms into a single histogram, we obtain the final multiresolution regional face descriptor presented in Equation 4.

$$
F_{j}=\left[\begin{array}{llll}
H_{P, 1, j}^{\mu 2} & H_{P, 2, j}^{\mu 2} & \ldots . . & H_{P, R, j}^{\mu 2}
\end{array}\right]
$$

This regional facial descriptor can be used to measure the face similarity by summing the similarities between all the regional histograms. However, by directly applying the similarity measurement to the multi-scale LBP histogram [10], the performance will be compromised. The reason is that this histogram is of high dimensionality and contains redundant information. By adopting the idea from [7], the dimension of the descriptor can be reduced by employing principal component analysis (PCA) before LDA. PCA is used to extract the statistically independent information as a basis for LDA to derive discriminative facial features. Thus a regional discriminative facial descriptor, $D_{j}$, is defined by projecting the histogram information, $\mathrm{F}_{\mathrm{j}}$, into LDA space $\mathrm{W}_{\mathrm{j}}^{\text {lda }}$, i.e.

$$
D_{j}=\left(W_{j}^{l d a}\right)^{T} F_{j}
$$

After the projection, the similarity measurement presented below is obtained by summing the similarity, i.e. normalized correlation, of regional discriminative descriptors. 


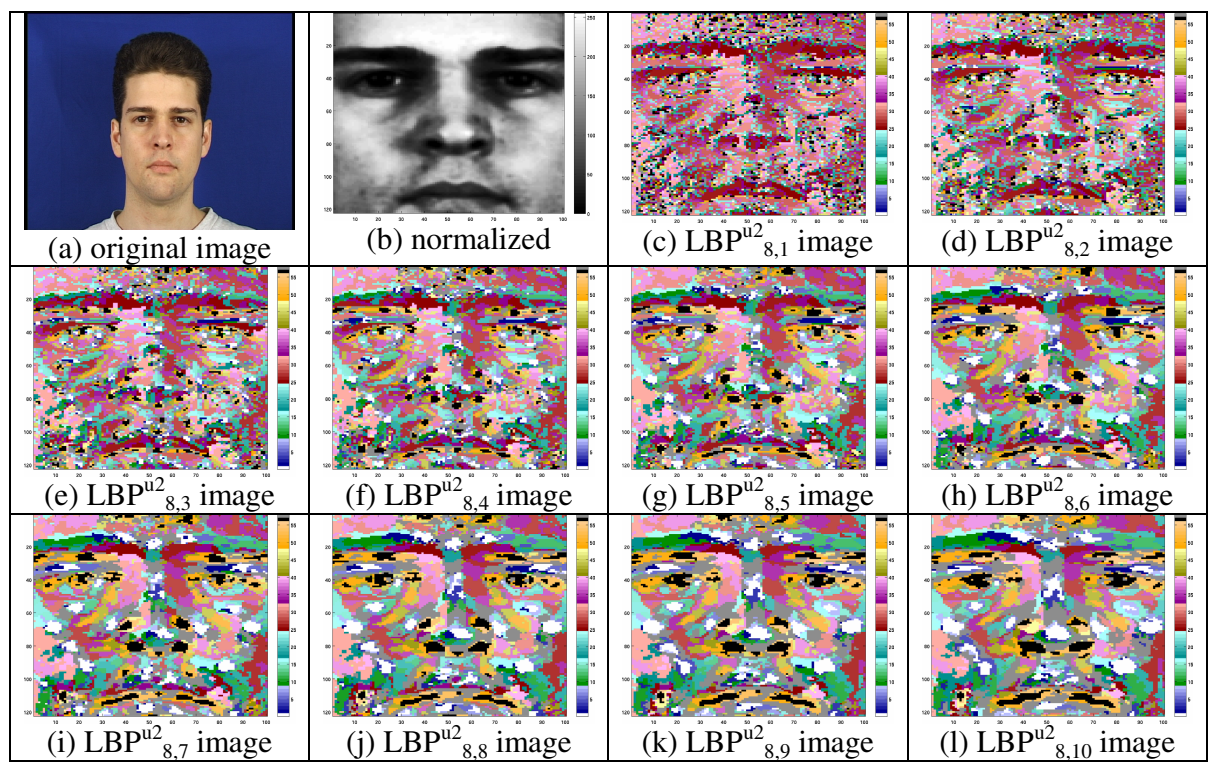

Fig. 1. a) original image, b) cropped and normalized face image, c-1) $\mathrm{LBP}^{\mathrm{u} 2}$ images at different radii. (Note: Gray: non-uniform pattern, White: dark spot, Black: bright spot, Other colors: rotational uniform patterns where 8 brightness levels of color denote the rotational angle).

$$
\operatorname{Sim}\left(I, I^{\prime}\right)=\sum_{j} \frac{D_{j} D_{j}{ }^{\prime}}{\left\|D_{j}\right\| D_{j}{ }^{\prime} \|}
$$

This discriminative descriptor gives 4 different levels of locality: 1) the local binary patterns contributing to the histogram contain information at the pixel level, 2) the patterns at each scale are summed over a small region to provide information at a regional level, 3) the regional histograms at different scales are concatenated to produce multiresolution information, 4) the global description of face is established by concatenating the regional discriminative facial descriptors.

\section{Experimental Setup}

The goals of identification and verification systems are different. Whereas the goal of identification is to recognize an unknown face image, verification validates a person's identity by comparing the captured face image with her/his image template(s) stored in the system database. However, most researchers only evaluate their algorithm either in identification or verification scenario, which makes them very difficult to compare with others. In order to ensure a reproducibility of the experiments and comparability with other methods, we tested our approach on the well-known, FERET and XM2VTS, databases using common protocols.

In the FERET database [6], the open-source publicly available CSU face identification evaluation framework [3] was utilized to test the performance of our 
method. In this experiment, only frontal faces are considered. They are divided into a standard gallery (fa set) containing 1196 images of 1196 subjects, and four probe sets, namely the fb set (1195 images containing different facial expressions), fc set (194 images acquired under different lighting conditions), dup I set (722 images taken a week later), dup II set (234 images taken at least a year later). The CSU standard training set containing 510 images from fa set and dup I set are used for computing the LDA transformation matrix, $\mathrm{W}_{\mathrm{j}}^{\text {lda }}$.

The XM2VTS frontal face database [4] contains 2360 images of 295 subjects, captured for verification over 4 sessions in a controlled environment. The testing is performed using the Lausanne protocol which splits the database into training, evaluation and test sets. The training set has 200 subjects as clients, the evaluation set contains additional 25 subjects as imposters and the testing set another 70 subjects as imposters. There are two configurations of the Lausanne Protocol. In our work, we use Configuration I, in which the client images for training and evaluation were acquired from the first three sessions. The decision of acceptance or rejection is based on a measurement of similarity between the gallery and the average of client's training images with a global threshold. This threshold is selected at the equal error point, EER, at which the false rejection rate is equal to the false acceptance rate on the evaluation set. For both XM2VTS and Feret databases, face images are extracted with the provided groundtruth eye positions and scaled to a size of $142 \times 120$ (rows $\times$ columns). The cropped faces are photometrically normalized by histogram equalization.

In total, four parameters are available to optimize the performance of our method. The first one is the LBP parameter, the circularly symmetric neighborhood size, P. A large neighborhood increases the length of the histogram and slows down the computation of the similarity measure while small neighborhood may result in information loss. We have selected a neighborhood of $\mathrm{P}=8$, containing 59 patterns for $\mathrm{LBP}^{\mathrm{u} 2}$. The second parameter is the total number of multi-scale operators. A small number of operators cannot provide sufficient information for face recognition, while a large radius operator not only reduces the size of the corresponding LBP images, but also decreases the number of uniform patterns which tends to degrade the system accuracy. In our experiments, $\mathrm{R}$ is set to 10 , which means that ten LBP operators are employed to represent the face image. After extracting the LBP images, they are then cropped to the same size. The third parameter is the number of the regions, $\mathrm{k}$. A large number of small regions increases the computation time as well as degrading the system accuracy in the presence of face localization errors. A big region increases the loss of spatial information. In this work, an image is partitioned into $\mathrm{k} \times \mathrm{k}$ nonoverlapped rectangle size regions where $\mathrm{k}$ is optimized empirically. The last parameter controls the PCA transformation matrix. In general, some of the higherorder eigenvectors are removed because they do not contribute to the accuracy of face recognition and the measure also saves the computation time. In our experiments, the number of eigenvectors kept is determined by the requirement to retain $98 \%$ of the energy of the signal [3]. 


\section{Results and Discussions}

\subsection{Experiments in Face Identification: FERET Database}

In this test, the recognition rate at rank1 and two statistical measures are used to compare the performance of the methods. The measures are the mean recognition rate with $95 \%$ confidence interval and the probability of the algorithm outperforming another. The probability is denoted by $\mathrm{P}(\mathrm{Alg} 1>\operatorname{Alg} 2)$. These measures are computed by permuting the gallery and probe sets, see [3] for details. The results with PCA, BIC and EBGM in the CSU system as benchmarks [3] are reported in Table 1 for comparison.

The result of the $\mathrm{LBP}_{8,2}^{\mathrm{u} 2}$ regional histograms method with chi-squared similarity measurement (LBPH_Chi) [9], $\mathrm{LBP}^{\mathrm{u} 2}{ }_{8,2}$ regional histograms projected on LDA space with normalized correlation (LBPH+LDA) and our proposed method $(\mathrm{MLBPH}+\mathrm{LDA})$ with different $\mathrm{k} \times \mathrm{k}$ regions are plotted in Fig. 2. Comparing the mean recognition rate with LBPH_Chi and LBPH+LDA, applying LDA to the representation generated by uniform pattern regional histograms clearly improves the performance, but employing the multi-scale LBP improves the recognition rate even further. As expected for the LBP histogram based methods, the mean recognition rate is reduced as the window size increases because of the loss of the spatial information, but for our method, the mean recognition rate is robust for a wide range of $16 \geq k>3$

Table 1. Comparisons on the probe sets and the mean recognition rate of the permutation test with 95\% confidence interval on the FERET database with CSU Standard training set

\begin{tabular}{|l|l|l|l|l|l||l|l|l|}
\hline & $\mathrm{k}$ & $\mathrm{Fb}$ & Fc & Dup1 & Dup2 & Lower & Mean & Upper \\
\hline MLBPH+LDA & 11 & 0.986 & 0.711 & 0.722 & 0.474 & 0.844 & 0.885 & 0.925 \\
\hline LBPH+LDA & 16 & 0.977 & 0.747 & 0.710 & 0.491 & 0.819 & 0.856 & 0.900 \\
\hline LBPH_Chi & 16 & 0.964 & 0.588 & 0.648 & 0.487 & 0.744 & 0.791 & 0.838 \\
\hline PCA_MacCos & & 0.853 & 0.655 & 0.443 & 0.218 & 0.662 & 0.721 & 0.775 \\
\hline Bayesian_MP & & 0.818 & 0.351 & 0.508 & 0.299 & 0.669 & 0.720 & 0.769 \\
\hline EBGM_Optimal & & 0.898 & 0.418 & 0.463 & 0.244 & 0.621 & 0.664 & 0.712 \\
\hline
\end{tabular}

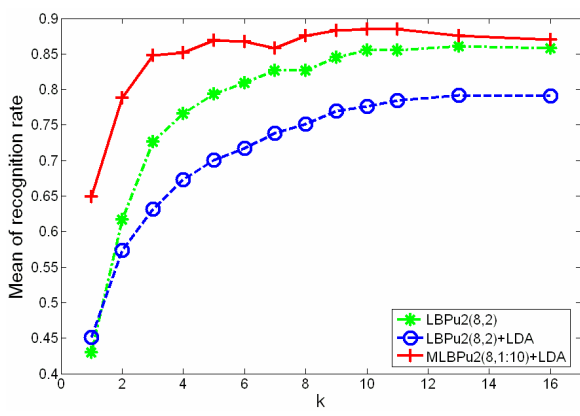

Fig. 2. The mean recognition rate with $95 \%$ confidence interval for three LBP methods against different $\mathrm{k} \times \mathrm{k}$ regions.

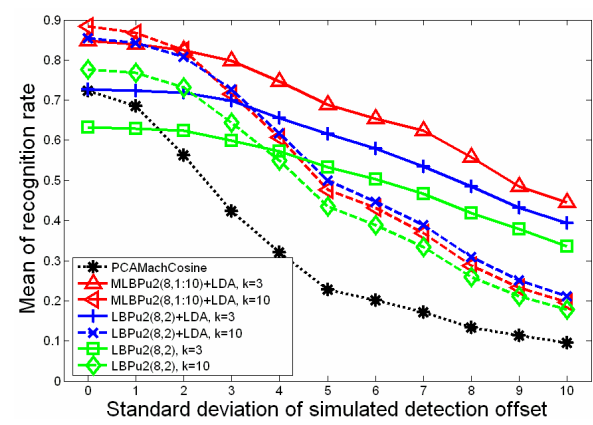

Fig. 3. The mean recognition rate with $95 \%$ confidence interval for LBP based methods and PCA MahCosine against varying the standard deviation of the simulated localization error. 
regions. For example the mean recognition rate with $\mathrm{k}=3$ is $84.8 \%$, while $\mathrm{k}=11$ is $88.5 \%$. In other words, changing the number of regions, $\mathrm{k}$, only affects the length of the feature vector and the computation time. In the presence of the face localization inaccuracies, the performance of the face recognition method involving spatial information as an input parameter degrades; however our proposed method using smaller $\mathrm{k}$ can be expected to maintain the recognition accuracy. These finding are discussed further in Section 4.2.

In Table 1, the parameter $\mathrm{k}$ of the LBP-based method is optimized from the point of view of accuracy and compared with other methods. LBP with LDA based methods clearly outperform the others in all statistical tests and all probe sets. Comparing MLBP and LBP both with LDA, the accuracy is not significantly different, but MLBPH+LDA is slightly better as $\mathrm{P}(M L B P H+L D A>L B P H+L D A)=0.898$.

\subsection{Robustness to Face Localization Error}

A generic face recognition system first localizes and segments a face image from the background before recognizing it. However, a perfect face localization method is very difficult to achieve, and therefore a face recognition method capable of working well in the presence of localization errors is highly desired. In order to evaluate the effect of face localization error on the recognition rate of our method achieved on the FERET database comparatively, PCA MachCosine, LBPH+LDA and LBPH+Chi face recognition methods are also implemented. The training images and gallery images, fa set, are registered using the groundtruth eye coordinates but the probe sets ( $\mathrm{fb}, \mathrm{fc}$, Dup 1 and 2) are registered using simulated eye coordinates which are the groundtruth eye location displaced by a random vector perturbation $(\Delta \mathrm{X}, \Delta \mathrm{Y})$. These vectors are uncorrelated and normally distributed with a zero mean and standard deviation, $\sigma$, from 0 to 10. For LBP based methods, a large region size parameter, $\mathrm{k}=3$, and a small region size, $\mathrm{k}=10$, are tested. The recognition rates of LBP based methods using the respective values of parameter $\mathrm{k}$, with PCA MachCosine against the standard deviation of the simulated localization error are plotted in Fig. 3. Clearly, the recognition rates of local region based methods outperform that of PCA. Projecting LBP histograms on LDA spaces provides better recognition rate than the error achieved in the original histogram space, in spite of the localization error. Also, for the local region based histogram methods, the larger region size the better the recognition rate as the localization error increases. Most importantly, in the presence of localization error, the recognition rate of MLBPH+LDA using a larger window size is more robust than others. The main reasons for the superior performance are the combination of the histogram approach and the multiresolution representation.

\subsection{Experiments in Face Verification: XM2VTS Database}

In verification tests, the total error, TER, which is the summation of the false rejection rate and the false acceptance rate, is used to report the performance of the methods. In this experiment, we compare $\mathrm{LBP}_{8,2}^{\mathrm{u} 2}$ with Chi-squared (LBPH_Chi), histogram intersection (LBPH_HI), and our proposed method (MLBPH+LDA) together with the Adaboost classifier for LBPH [2] (LBPH+Adaboost). Rodriguez [14] found that the total error rate of $\mathrm{LBPH}-A d a b o o s t$ giving $7.88 \%$ on the test set, is similar to that of LBPH-Chi, namely $6.8 \%$. Nevertheless, we found that the error rate of LBPHAdaboost can be reduced to $5.263 \%$ if 300 regional histograms (features) are used. 
Table 2. Total Error Rate, TER, according to Lausanne protocol for configuration 1

\begin{tabular}{|l|c|c|c||c|c|}
\hline & $\mathrm{k}$ & \multicolumn{2}{|c||}{ Manual Registration } & \multicolumn{2}{c|}{ Automatic Registration } \\
\hline & & Eva Set (\%) & Test Set (\%) & Eva Set (\%) & Test Set (\%) \\
\hline MLBPH+LDA & 3 & 1.74 & 1.48 & 1.53 & 1.99 \\
\hline LBPH+LDA & 6 & 8.39 & 6.74 & & \\
\hline LBPH_Chi [9] & 7 & 11.11 & 8.27 & & \\
\hline LBPH_HI & 7 & 10.28 & 7.94 & & \\
\hline LBPH+AdaBoost [2] & & 7.37 & 5.26 & & \\
\hline LBPH_MAP [14] & & & 2.84 & & \\
\hline LBP+LDA [1] & & & 9.12 & & \\
\hline LBP+HMM [1] & & & 2.74 & & 13.10 \\
\hline ICPR2000-Best [5] & & 5.00 & 4.80 & 14.00 & 3.86 \\
\hline AVBPA03-Best [5] & & 2.21 & 1.47 & 4.98 & $2.07^{2}$ \\
\hline ICB2006-Best [5] & & 1.63 & 0.96 & 2 & \\
\hline
\end{tabular}

Table 2 reports the comparative results of the above mentioned methods, as well as of Rodriguez methods [14][1], and the performance of the best ICPR2000[5], the best AVBPA2003[5] and the best ICB2006[5] algorithms with the Lausanne protocol Configuration 1.

Compared to other LBP based methods, it is clear that our proposed method, MLBPH+LDA, performs better. However, the result of our method in manual registration is not better than that in ICB2006, in which the features were extracted by convoluting the gabor filters, 5 scales and 8 orientation, on the face image. Since the method in ICB2006 uses face images of better resolution than ours, we can expect that the face manifolds of the gabor feature space are simpler and the associated error rate is lower. Nevertheless, our method is more robust than others in the presence of face localization inaccuracies as shown in Table 2.

\section{Conclusions}

In a real face recognition system, a face image is detected, registered and then identified. However, the accuracy of automatic face localization is not perfect and therefore face recognition methods working successfully in the presence of localization error are highly desired. In this paper, a discriminative descriptor containing the information from a multiresolution analysis of face image is proposed. The descriptor is formed by projecting the local face image information acquired by multiple LBP operators, into the LDA space. The recognition is performed by measuring the dissimilarity of the gallery and probe descriptors. Our proposed method has been implemented and compared with existing LBP methods as well ass other well known benchmarks in the application of face identification and verification using the FERET and XM2VTS databases following their standard protocols. In face identification performed on the FERET database, the experimental results clearly show that the mean recognition rate of $88.5 \%$, with a $95 \%$ confidence interval, delivered by our method outperforms other state-of-the-art contenders. In particular, our method achieved the overall best result of $98.6 \%$ recognition rate in the

\footnotetext{
${ }^{2}$ Note: There is a mistake in the TER of the best algorithm reported in ICB 2006, where TER, 1.57, is not equal to the summation of false acceptance rate, 0.57 and the false rejection rate, 1.57.
} 
experiment involving the varying facial expression probe set ( $\mathrm{fb}$ set) while delivering comparative results to other LBP based methods for other probe sets. Also under the simulated localization error test, our proposed method is clearly more robust than others because it benefits from the multiresolution information captured by the regional histograms. The proposed method has also been tested in the verification mode on the XM2VTS database. With manual registration it achieved the third best result, TER $=1.48 \%$ on the test set, but with fully automatic registration outperformed all the other methods by a small margin, achieving, TER $=1.99 \%$. In conclusion, our method achieves a comparable result with the state-of-art benchmark methods, on manually annotated face but it is more robust in the presence of localization errors.

\section{Acknowledgements}

A partial support from the EU Network of Excellence Biosecure and from the ESPRC Grant GR/S98528/01 is gratefully acknowledged.

\section{References}

1. Heusch, G., Rodriguez, Y., Marcel, S.: Local Binary Patterns as an Image Preprocessing for Face Authentication. In: Proc FGR 2006, pp. 9-14 (2006)

2. Zhang, G., Huang, Z., Li, S.Z.: Boosting local binary pattern (LBP)-based face recognition. In: SinoBiometrics 2004, pp. 179-186 (2004)

3. Beveridge, J.R., Bolme, D., Teixeira, M., Draper, B.: The CSU Face Identification Evaluation System User's Guide: Version 5.0, Technical Report, C.S. Dept., CSU (2003)

4. Messer, K., Matas, J., Kittler, J., Jonsson, K.: XM2VTSDB: The extended M2VTS database. In: Akumuri, S., Kullman, C. (eds.) AVBPA’99, pp. 72-77 (1999)

5. Messor, K., Kittler, J., Short, J., Heusch, G., Cardinaux, F., Marcel, S., Rodriguez, Y., Shan, S., Su, Y., Gao, W., Chen, X.: Performance Characterisation of Face Recognition Algorithms and Their Sensitivity to Severe Illumination Changes. In: ICB2006, pp. 1-11 (2006)

6. Philips, P.J., Moon, H.J., Rizvi, S.A., Rauss, P.J.: The FERET Evaluation Methodology for Face Recognition Algorithms. PAMI 22(10), 1090-1104 (2000)

7. Belhumeur, P.N., Hespanha, J.P., Kriegman, D.J.: Eigenfaces vs. Fisherfaces: Recognition using Class Specific Linear Projection. In: Buxton, B.F., Cipolla, R. (eds.) ECCV 1996. LNCS, vol. 1065, pp. 45-56. Springer, Heidelberg (1996)

8. Shan, S., Zhang, W., Su, Y., Xhen, X., Gao, W.: Ensemble of Piecewise FDA based on Spatial Histogram of Local (Gabor) Binary Patterns for Face Recognition. In: ICPR (2006)

9. Ahonen, T., Hadid, A., Pietikäinen, M.: Face description with local binary patterns: Application to face recognition. PAMI 28(12), 2037-2041 (2006)

10. Ojala, T., Pietikäinen, M., Mäenpää, T.: Multiresolution Gray-Scale and Rotation Invariant Texture Classification with Local Binary Patterns. PAMI 24(7), 971-987 (2002)

11. Zhang, W., Shan, S., Zhang, H., Gao, W., Chen, X.: Multi-resolution histogram of local variation patterns (MHLVP) for Robust Face Recognition. In: Kanade, T., Jain, A., Ratha, N.K. (eds.) AVBPA 2005. LNCS, vol. 3546, pp. 937-944. Springer, Heidelberg (2005)

12. Huang, X., Li, S.Z., Wang, Y.: Jensen-Shannon Boosting Learning for Object recognition. In: Proceeding of CVPR (2005)

13. Raja, Y., Gong, S.: Sparse Multiscale Local Binary Patterns. In: Proc. $17^{\text {th }}$ BMVC (2006)

14. Rodriguez, Y., Marcel, S.: Face authentication using adapted local binary pattern histogram. In: Leonardis, A., Bischof, H., Pinz, A. (eds.) ECCV 2006. LNCS, vol. 3954, pp. 321-332. Springer, Heidelberg (2006) 